\title{
Polymyositis Presenting as Rhabdomyolysis After the Initiation of Omeprazole
}

\author{
Jonathan K. Jakubowski ${ }^{1}$, Rosemina Patel ${ }^{1}$, Venkata Buddharaju ${ }^{2}$
}

1. Internal Medicine, Chicago Medical School - Rosalind Franklin University of Medicine and Science, North Chicago, USA 2. Nephrology, Chicago Medical School - Rosalind Franklin University of Medicine and Science, North Chicago, USA

Corresponding author: Jonathan K. Jakubowski, jonathan.jakubowski@rosalindfranklin.edu

\begin{abstract}
Rhabdomyolysis is a clinical syndrome with a wide range of presentations; it results in muscle necrosis and release of intracellular muscle contents into the circulation. Inflammatory myopathies are a rare cause of rhabdomyolysis. We present a case of a 46-year-old male with a two-week history of progressively worsening diffuse muscle pain after he had been prescribed omeprazole one month prior. A creatine phosphokinase (CPK) elevation was noted, which persisted despite treatment with IV fluids, sodium bicarbonate, and close correction of electrolytes. Further workup, including autoimmune and infectious etiologies, was notable for elevated antinuclear antibodies (ANA), erythrocyte sedimentation rate (ESR), and C-reactive protein (CRP). Furthermore, a muscle biopsy showed evidence of endomysial inflammatory cells, consistent with a diagnosis of polymyositis. Steroids were initiated with significant improvement in symptoms and a decrease in CPK levels. The patient was discharged on a tapering dose of steroids and, on follow-up with the rheumatologist, transitioned to methotrexate with control of symptoms. In patients with rhabdomyolysis who do not respond to first-line therapy, obtaining a detailed medication history and screening with ANA and ESR are encouraged. Given the link between medication and autoimmune disease, clinicians should consider autoimmune myopathy in the differential for cases with persistently elevated creatine kinase. Prompt diagnosis with early initiation of immunosuppressive medication may improve outcomes and avoid complications associated with untreated rhabdomyolysis or polymyositis.
\end{abstract}

Categories: Nephrology, Rheumatology

Keywords: rhabdomyolysis, polymyositis, proton pump inhibitor

\section{Introduction}

Rhabdomyolysis is a clinical syndrome that results in muscle necrosis and the release of muscle cell contents into the circulation, most notably myoglobin. Rhabdomyolysis is associated with a wide-spectrum manifestation, from remaining clinically silent as a benign course to a severe systemic presentation causing pigment-induced nephropathy [1]. It may arise from a traumatic or non-traumatic etiology including toxins, electrolyte disturbances, infection, medications, immobilization, seizures, and, rarely, autoimmune myopathies. Medications such as statins have been documented to contribute to the development of autoimmune myopathies [2-4]. However, only a few cases of proton pump inhibitor (PPI)-induced myopathies have been reported. Inflammatory myopathies are a rare cause of rhabdomyolysis. We present a unique case of a patient who initially presented with rhabdomyolysis, later with hemoptysis, and was eventually diagnosed with polymyositis.

\section{Case Presentation}

A 46-year-old Hispanic male presented in late summer with three days of abdominal pain and diarrhea. He also endorsed a two-week history of progressively worsening diffuse muscle pain, notably worse in the lower extremities. He denied any trauma, recent illness, or any relevant family medical history. His medical history included gastroesophageal reflux disease diagnosed one month ago, for which omeprazole had been prescribed, which had led to an improvement of his heartburn. On examination, vital signs were within normal limits and he had mild tenderness to palpation of the abdomen. Extremities showed decreased muscle strength, which was more profound in the lower extremities; however, he remained neurologically intact. Initial labs showed aspartate aminotransferase (AST) of 494, alanine aminotransferase (ALT) 290, troponin I of 0.36 , creatine kinase-MB (CKMB) 915.5 with a relative index of 11.5 , and a creatine phosphokinase (CPK) of 7974. Urine dipstick was positive for blood; however, no RBCs were seen on microscopy. A urine drug screen was negative. His electrocardiogram showed normal sinus rhythm with no ST-T wave changes. A CT of the abdomen was obtained, which was unremarkable.

The patient was admitted and started on aggressive IV fluids for rhabdomyolysis and non-ST elevated myocardial infarction (NSTEMI). His home medication was held on admission. To rule out acute coronary syndrome, the patient underwent a cardiac workup with an echocardiogram, which showed a normal ejection fraction and no wall motion abnormalities; he also underwent a nuclear stress test later, which was negative for myocardial ischemia. Elevated troponin was therefore suggested to be related to 


\section{Cureus}

rhabdomyolysis. The patient was still symptomatic with myalgia and CPK remained elevated above 6000 despite adequate hydration and addition of a bicarbonate infusion. On hospital day six, the patient underwent further evaluation for the persistent elevation of CPK. Infectious workup including hepatitis A, B, and $C$ returned negative. ANA was noted to be greater than 1:640 with a speckled pattern; CRP of 2.83 and ESR of 44 were also observed. An autoimmune cause for rhabdomyolysis was suspected. A trial of steroids with methylprednisolone $40 \mathrm{mg}$ IV was given, with remarkable improvement of symptoms. The patient's CPK declined to 4000 , and he was discharged on a tapering dose of prednisone for suspected autoimmune myositis. The patient returned less than 24 hours later with a similar presentation with a new onset of hemoptysis.

During the second admission, he was given $1 \mathrm{mg} / \mathrm{kg}$ of IV methylprednisolone. Omeprazole was again held on admission with a transition to famotidine. Repeat laboratory data showed a CPK of 5026, serum aldolase of 81.3, and urine dipstick positive for blood; however, no red blood cells on microscopy were observed. CT angiogram of the chest was obtained, which was negative for pulmonary embolism but showed bilateral parenchymal nodular opacities (Figure 1,2). The patient underwent bronchoscopy, which showed normal endobronchial anatomy without gross hemorrhage or hemoptysis, and with cytopathology showing hemosiderin-laden macrophages, consistent with diffuse alveolar hemorrhage (DAH). A complete serologic panel was obtained, which came back negative (Table 1). For a definitive diagnosis of inflammatory myositis, the patient underwent a muscle and skin biopsy of the right quadriceps. The skin biopsy showed no significant histological abnormalities; however, the muscle biopsy showed evidence of endomysial inflammatory cells in the muscle fascicles with regeneration, consistent with a diagnosis of polymyositis. There was no evidence of necrosis, rimmed vacuoles, or perivascular inflammatory changes. High- dose steroids were continued with a resolution of hemoptysis and muscle pain. CPK continued to trend down with no further evidence of myoglobinuria (Figure 3). Omeprazole was discontinued indefinitely. He was discharged on a tapering dose of oral prednisone.

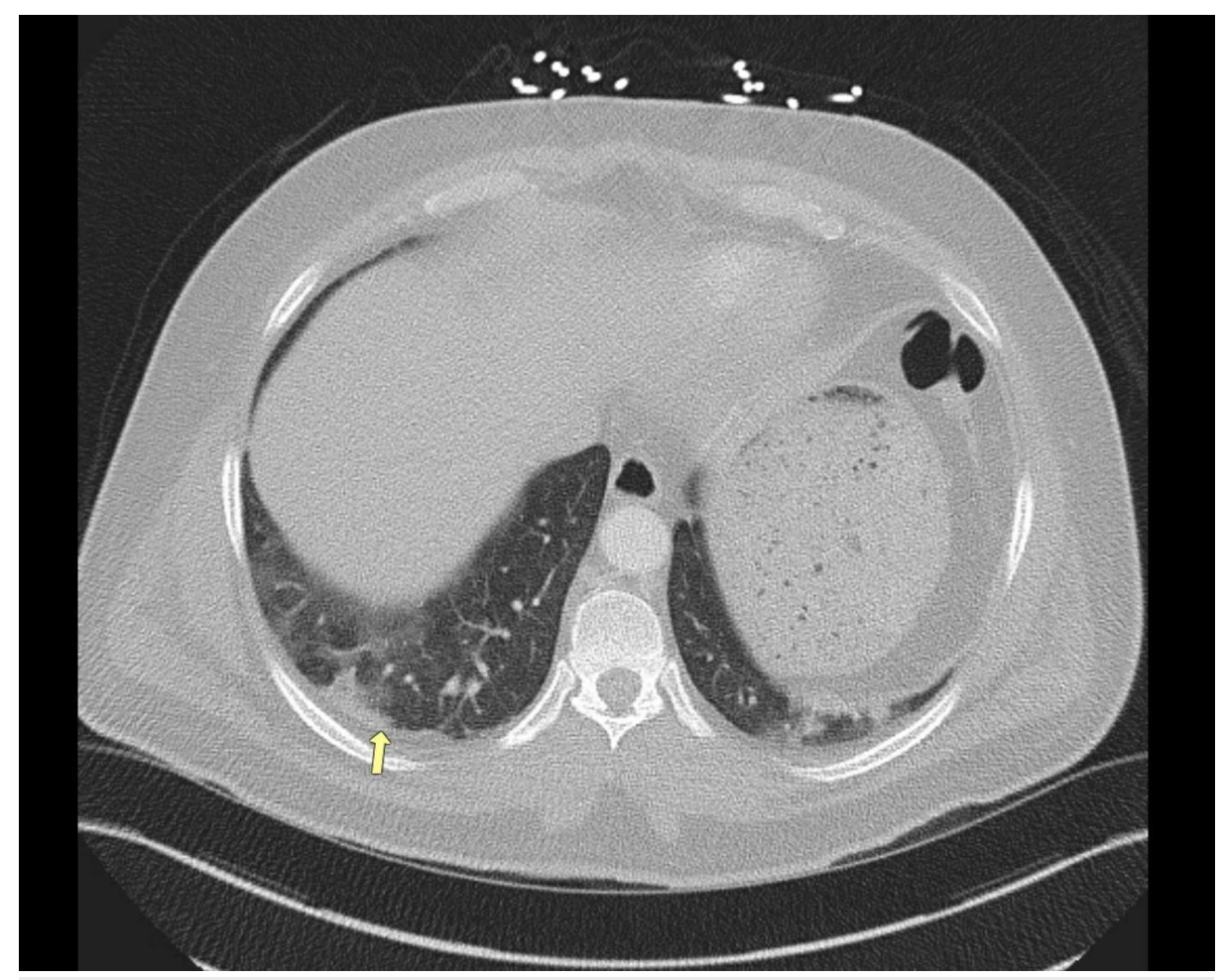

FIGURE 1: CT angiogram of the chest, with nodular opacities (arrow)

CT: computed tomography 


\section{Cureus}

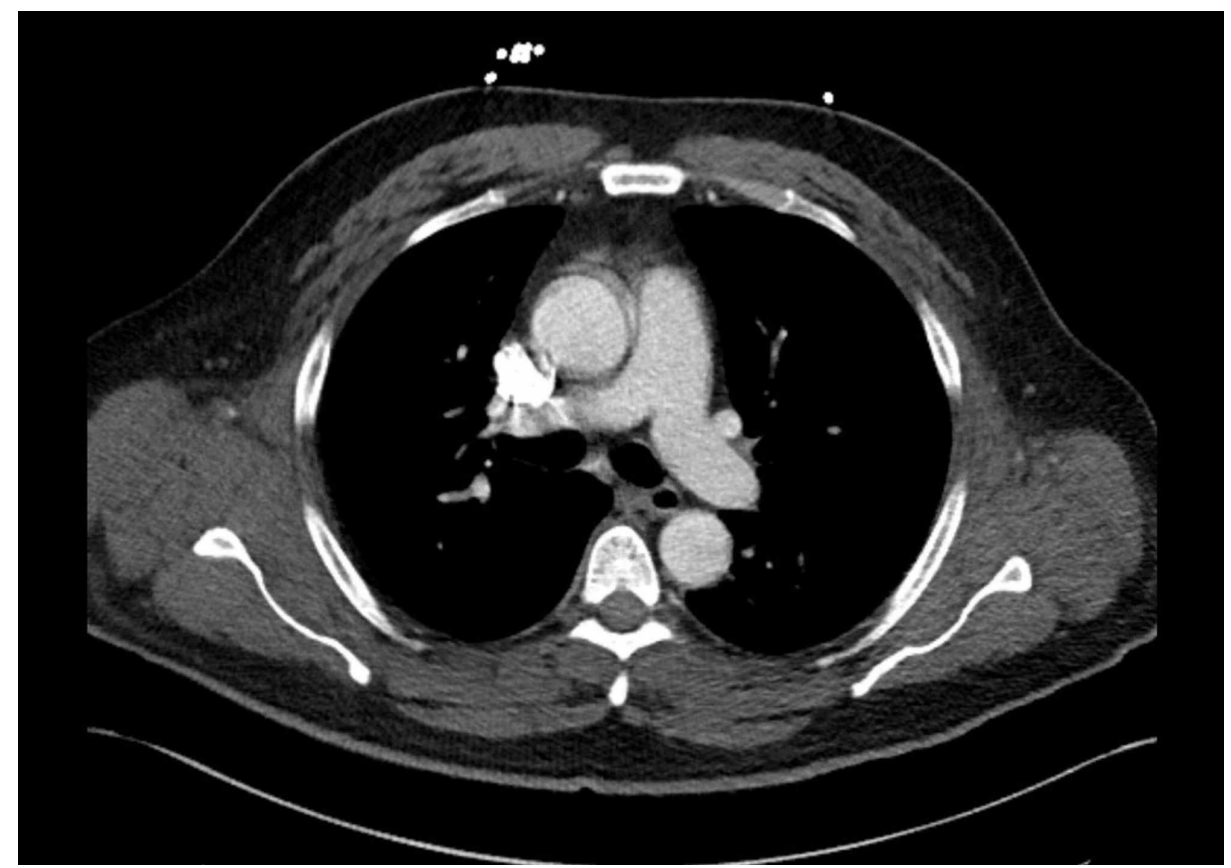

FIGURE 2: CT angiogram of the chest

The image shows no evidence of pulmonary embolism from the main pulmonary artery through the bifurcation to the right and left pulmonary arteries

CT: computed tomography

\begin{tabular}{|l|l|l|}
\hline Antibody & Result & Reference range \\
\hline ANA titer & $>1: 640$ & \\
ANA pattern & Speckled & $0-40 \mathrm{AU} / \mathrm{mL}$ \\
Anti-U1 RNP & 5 & $0-40 \mathrm{AU} / \mathrm{mL}$ \\
Anti-SSA 52 (Ro) & 9 & $0-40 \mathrm{AU} / \mathrm{mL}$ \\
\hline Anti-Jo 1 & 3 & \\
\hline Anti-U3 RNP & Negative & $0-19 \mathrm{AU} / \mathrm{mL}$ \\
Anti-GBM & 0 & $0-40 \mathrm{AU} / \mathrm{mL}$ \\
Anti-SCL-70 & 8 & \\
\hline Anti-dsDNA & Negative & \\
ANCA IFA & $<1: 20$ & \\
\hline
\end{tabular}

\section{TABLE 1: Autoimmune workup of the patient}

ANA: antinuclear antibody; Anti-U1 RNP: anti-U1 ribonucleoprotein; Anti-SSA 52 (Ro): anti-Sjogren's syndrome-related antigen A/anti-Ro; Anti-U3 RNP: anti-U3 ribonucleoprotein; Anti-GBM: anti-glomerular basement membrane; Anti-SCL-70: anti-topoisomerase I; Anti-dsDNA: anti-doublestranded DNA; ANCA: anti-neutrophil cytoplasmic antibody; IFA: immunofluorescence assay 


\section{Cureus}

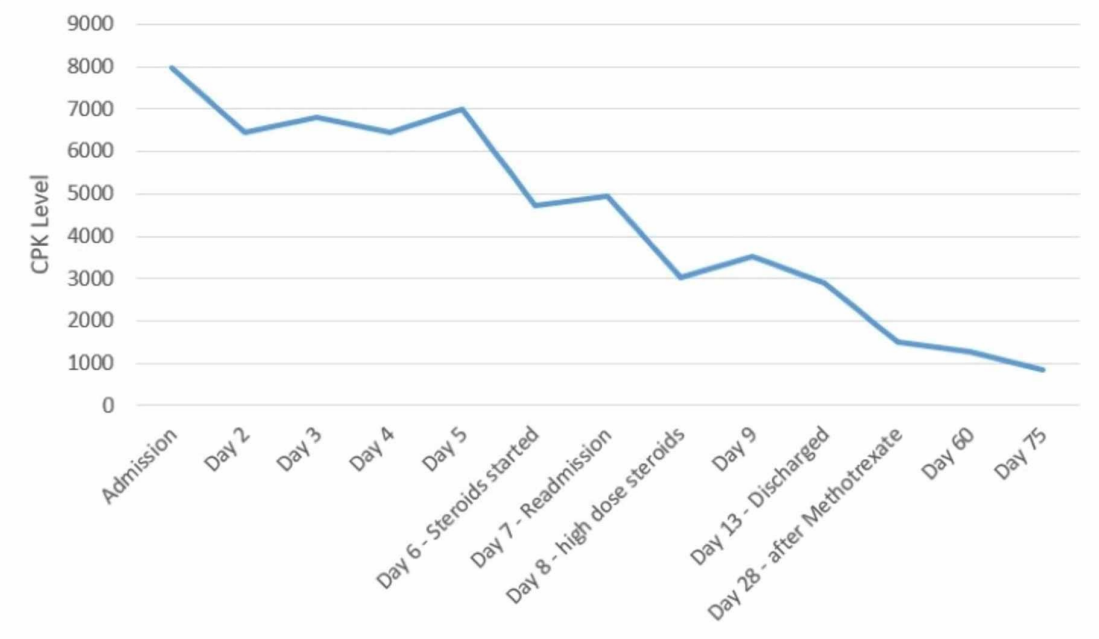

FIGURE 3: Muscle enzyme trends - from diagnosis to resolution

CPK: creatine phosphokinase

After discharge, the patient had an MRI of the left shoulder without contrast, which demonstrated a diffuse increase in T2 signal of the cuff muscles, suggestive of edema, and possibly active inflammation (Figure 4). He was seen by a rheumatologist who started him on methotrexate. The patient's symptoms remained well controlled after dose titration. A repeat CPK two months later was noted to be 1271 with other muscle enzymes also showing a downward trend.

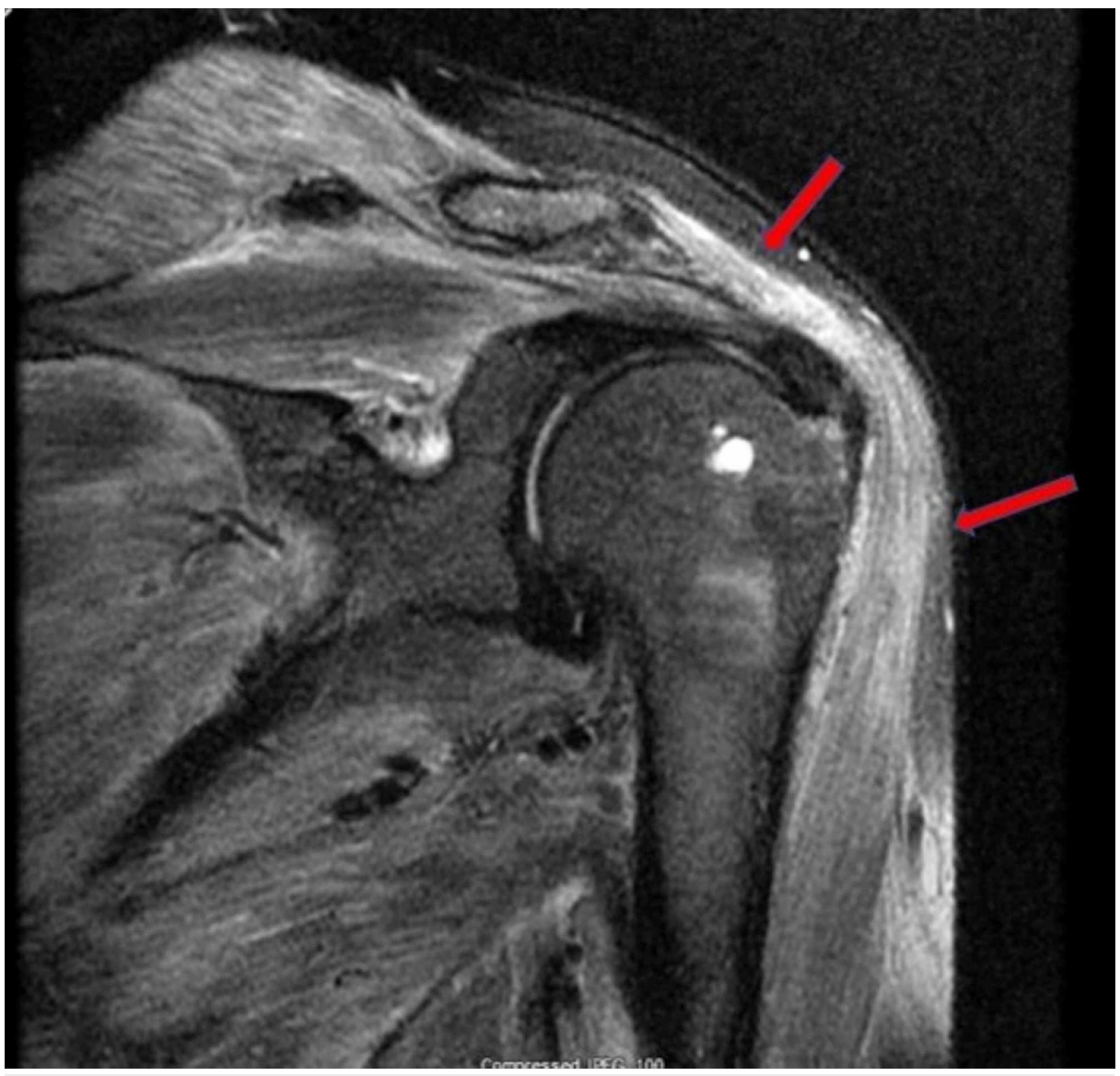

FIGURE 4: MRI of the left shoulder without contrast

The image shows diffuse muscle edema on T2-weighted image (arrows) 
Discussion

Polymyositis is a subtype of idiopathic inflammatory myopathy (IIM). The main symptom of the condition is symmetric proximal muscle weakness developing over three to six months. Other findings include elevation in muscle enzymes, muscle-specific antibodies, muscle biopsy showing endomysial inflammation with infiltrate rich in CD8 T cells, and increased major histocompatibility complex class I (MHC-1) expression. Diagnostic methods that aid in the diagnosis of polymyositis consist of electromyography indicating myopathy and MRI with T2-weighted images with fat suppression to identify edema. Our patient met three out of the four criteria in the Bohan and Peter classification for polymyositis, indicating probable polymyositis [5]. According to the European League Against Rheumatism and the American College of Rheumatology EULAR/ACR classification criteria for IIM, our patient had a score of 6.5, which is below the cut-off for the diagnosis of IIM; however, it should be kept in mind that the score does not include MRI and electromyography (EMG) data [6]. Our patient had MRI evidence of proximal muscle inflammation, which would have increased the probability of IIM.

Polymyositis is a rare cause of rhabdomyolysis as it typically presents insidiously. One retrospective study by Melli et al. reviewed 475 patients with rhabdomyolysis, of which only 27 patients were identified to have IIM (21 patients with polymyositis, four with dermatomyositis, and two with overlap syndrome) [7]. Individual case reports have been published in literature where IIM presented with rhabdomyolysis, as seen with our patient [8-10].

There is also evidence of PPIs increasing the risk of rhabdomyolysis in a few case series and reports. A review of individual case reports by Duncan and Howden identified seven cases of rhabdomyolysis associated with PPI. Two of these seven patients were taking HMG-CoA reductase inhibitors, which are known to cause varying degrees of myopathy. None of the patients were re-challenged with PPIs [11]. Hence, there is some evidence linking PPIs to rhabdomyolysis, although a causal relationship cannot be established. Another review study published by Clark et al. analyzed 35 cases of rhabdomyolysis associated with PPI treatment; 12 of these patients were concomitantly taking HMG-CoA reductase inhibitors. PPIs were re-introduced in only one patient in whom rhabdomyolysis did not re-occur. In the same study, there were 27 case reports in which PPI was associated with an IIM [12]. Of those cases, six were confirmed polymyositis, five were related to omeprazole, with one due to pantoprazole. In about $45 \%$ of the reports, where time to onset was given, the symptoms developed during the first three months of treatment.

Several mechanisms have been proposed for PPIs as a cause of rhabdomyolysis. PPIs are metabolized through the hepatic cytochrome P450 enzymes, with CYP2C19 having the primary role with inhibition of CYP3A4 to some extent. Many other drugs, like statins, undergo metabolism by CYP3A4. These pharmacokinetic interactions can increase exposure to statins, thus increasing the risk of rhabdomyolysis. CYP2C19 polymorphisms contribute to the reduced metabolism of PPIs [13]. A study by Goktas et al. showed that the CYP2C19 activity of the patients with Behçet's disease was significantly lower as compared to healthy subjects [14]. PPIs act by binding to $\mathrm{H}+/ \mathrm{K}+-\mathrm{ATPase}$ in gastric parietal cells, and theoretically may also bind to other cells like vascular smooth muscle cells, which can alter the intracellular environment and predispose cells to degrade. Another proposed mechanism may be related to decreased metabolism of reactive oxygen species (ROS). A study by May et al. showed reduced cytochrome P-450 activity causing a decline in ROS metabolism, which may play a significant role in scleroderma pathogenesis [15]. Previous studies had found that pathogenesis of autoimmune diseases might be related to increased ROS [15]. Another study by Sivakumar and Dalakas provided evidence that autoimmune disease due to omeprazole use may be caused by an alteration in the catabolism of nuclear chromatin by omeprazole, similar to what has been proposed as a cause of drug-induced lupus [16].

Given our patient's presentation after initiating omeprazole, we now believe he may have had a CYP2C19 polymorphism, which triggered the development of polymyositis, although we were unable to prove it at the time. Further data are needed to confirm our suspicion. Given the link between PPIs and autoimmune disease, we suggest keeping a high index of suspicion for inflammatory myopathies by obtaining a detailed medication history and screening with an ANA and inflammatory markers in patients with rhabdomyolysis who do not respond to first-line therapy.

It is unclear why our patient developed alveolar hemorrhage. DAH in autoimmune disorders is usually seen in systemic vasculitis syndromes, mixed connective tissue disorders (MCTD), and anti-glomerular basement membrane antibodies. Our patient did not show any serological evidence of other systemic diseases, making an overlap syndrome less likely. While lung is the most common extra-muscular site of involvement in inflammatory myopathies, pulmonary manifestations usually include interstitial lung disease. Reports of DAH in IIMs, although rare, are found in the literature [17,18]. Most cases of DAH have been associated with increased mortality due to respiratory failure unless promptly treated with immunosuppressive therapy [19]. In patients who underwent an autopsy, biopsy showed pulmonary capillaritis [20]. Therefore, prompt treatment with methylprednisone and/or IV cyclophosphamide should be started in patients with 
inflammatory myopathies in whom DAH is present. Fortunately, our patient did not develop overt respiratory failure, probably due to being on prednisone therapy on the second presentation, and did not show any evidence of DAH on follow-up.

\section{Conclusions}

Clinicians should consider an autoimmune myopathy in the differential for patients with persistently elevated CPK who are on medication linked to the diagnosis. A prompt diagnosis followed by early initiation of immunosuppressive medication may improve outcomes and avoid complications associated with untreated rhabdomyolysis or polymyositis. Given that PPIs are easily accessible over the counter and are among the most frequently prescribed medications, more studies are needed to explore their relationship with autoimmune myopathies.

\section{Additional Information \\ Disclosures}

Human subjects: Consent was obtained by all participants in this study. Conflicts of interest: In compliance with the ICMJE uniform disclosure form, all authors declare the following: Payment/services info: All authors have declared that no financial support was received from any organization for the submitted work. Financial relationships: All authors have declared that they have no financial relationships at present or within the previous three years with any organizations that might have an interest in the submitted work. Other relationships: All authors have declared that there are no other relationships or activities that could appear to have influenced the submitted work.

\section{References}

1. Vanholder R, Sever MS, Erek E, Lameire N: Rhabdomyolysis. J Am Soc Nephrol. 2000, 11:1553-1561.

2. Young JB, Ghobrial II: Autoimmune statin-induced myopathy: a case report . J Community Hosp Intern Med Perspect. 2015, 5:28374. 10.3402/jchimp.v5.28374

3. Kanth R, Shah MS, Flores RM: Statin-associated polymyositis following omeprazole treatment. Clin Med Res. 2013, 11:91-95. 10.3121/cmr.2012.1110

4. Troyanov Y, Landon-Cardinal O, Fritzler MJ, et al.: Atorvastatin-induced necrotizing autoimmune myositis: an emerging dominant entity in patients with autoimmune myositis presenting with a pure polymyositis phenotype. Medicine (Baltimore). 2017, 96:e5694. 10.1097/MD.0000000000005694

5. Bohan A, Peter JB: Polymyositis and dermatomyositis (first of two parts) . N Engl J Med. 1975, 292:344-347. 10.1056/NEJM197502132920706

6. Lundberg IE, Tjärnlund A, Bottai M, et al.: 2017 European League Against Rheumatism/American College of Rheumatology classification criteria for adult and juvenile idiopathic inflammatory myopathies and their major subgroups. Ann Rheum Dis. 2017, 76:1955-1964. 10.1136/annrheumdis-2017-211468

7. Melli G, Chaudhry V, Cornblath DR: Rhabdomyolysis: an evaluation of 475 hospitalized patients . Medicine (Baltimore). 2005, 84:377-385. 10.1097/01.md.0000188565.48918.41

8. Caccamo DV, Keene CY, Durham J, Peven D: Fulminant rhabdomyolysis in a patient with dermatomyositis . Neurology. 1993, 43:844-845. 10.1212/wnl.43.4.844

9. Kim HW, Choi JR, Jang SJ, Chang YS, Bang BK, Park CW: Recurrent rhabdomyolysis and myoglobinuric acute renal failure in a patient with polymyositis. Nephrol Dial Transplant. 2005, 20:2255-2258. 10.1093/ndt/gfh950

10. Marisiddappa L, Desai AM, Kedlaya PG, Sathish R: Recurrent episodic myoglobinuric acute kidney injury as presenting manifestation of idiopathic polymyositis. Saudi J Kidney Dis Transpl. 2018, 29:210-213. 10.4103/1319-2442.225181

11. Duncan SJ, Howden CW: Proton pump inhibitors and risk of rhabdomyolysis . Drug Saf. 2017, 40:61-64. 10.1007/s40264-016-0473-2

12. Clark DW, Strandell J: Myopathy including polymyositis: a likely class adverse effect of proton pump inhibitors?. Eur J Clin Pharmacol. 2006, 62:473-479. 10.1007/s00228-006-0131-1

13. Goldstein JA: Clinical relevance of genetic polymorphisms in the human CYP2C subfamily . Br J Clin Pharmacol. 2001, 52:349-355. 10.1046/j.0306-5251.2001.01499.x

14. Goktas MT, Karaca RO, Kalkisim S, et al.: Decreased activity and genetic polymorphisms of CYP2C19 in Behçet's disease. Basic Clin Pharmacol Toxicol. 2017, 121:266-271. 10.1111/bcpt.12710

15. May DG, Black CM, Olsen NJ, et al.: Scleroderma is associated with differences in individual routes of drug metabolism: a study with dapsone, debrisoquin, and mephenytoin. Clin Pharmacol Ther. 1990, 48:286-295. 10.1038/clpt.1990.151

16. Sivakumar K, Dalakas MC: Autoimmune syndrome induced by omeprazole. Lancet. 1994, 344:619-620. 10.1016/s0140-6736(94)92008-7

17. Sato K, Morozumi S, Takeuchi Y, Ochiai J, Mabuchi C: Case of dermatomyositis with anti-CADM-140 antibody and alveolar hemorrhage. (Article in Japanese). Rinsho Shinkeigaku. 2014, 54:408-412. 10.5692/clinicalneurol.54.408

18. Do-Pham G, Pagès C, Picard C, Galicier L, Lémann M, Dubertret L, Viguier M: A first case report of a patient with paraneoplastic dermatomyositis developing diffuse alveolar haemorrhage. Br J Dermatol. 2010, 163:227-228. 10.1111/j.1365-2133.2010.09800.x

19. Matsuki Y, Yamashita H, Takahashi Y, et al.: Diffuse alveolar damage in patients with dermatomyositis: a six-case series. Mod Rheumatol. 2012, 22:243-248. 10.1007/s10165-011-0502-Z

20. Schwarz MI, Sutarik JM, Nick JA, Leff JA, Emlen JW, Tuder RM: Pulmonary capillaritis and diffuse alveolar hemorrhage. A primary manifestation of polymyositis. Am J Respir Crit Care Med. 1995, 151:2037-2040. 


\section{Cureus}

10.1164/ajrccm.151.6.7767555 